\title{
Multidimensional poverty indices
}

\author{
Kai-yuen Tsui \\ Department of Economics, Chinese University of Hong Kong, Shatin, Hong Kong \\ (e-mail: b040785@mailserv.cuhk.edu.hk)
}

Received: 1 March 1999/Accepted: 1 August 2000

\begin{abstract}
This paper explores the axiomatic foundation of multidimensional poverty indices. Departing from the income approach which measures poverty by aggregating shortfalls of incomes from a pre-determined poverty-line income, a multidimensional index is a numerical representation of shortfalls of basic needs from some pre-specified minimum levels. The class of subgroup consistent poverty indices introduced by Foster and Shorrocks (1991) is generalized to the multidimensional context. New concepts necessary for the design of distribution-sensitive multidimensional poverty measures are introduced. Specific classes of subgroup consistent multidimensional poverty measures are derived based on sets of reasonable axioms. This paper also highlights the fact that domain restrictions may have a critical role in the design of multidimensional indices.
\end{abstract}

\section{Introduction}

The measurement of poverty has taken on a new lease of life thanks to Sen's pioneering article (Sen 1976). His well-known critique of the headcount and poverty-gap indices of poverty has stimulated a constant flow of academic interest on the axiomatic foundations and the design of poverty indices. ${ }^{1}$ Sen

I thank Professor A. Shorrocks for his comments on an earliear version of this paper. All remaining errors are mine. After finishing an earlier version of this paper which was started in 1993, I thank Professor Chakravarty for sending me two of his working papers (Bourguignon and Chakravarty 1997a,b) and the one with Mukherjee and Ranade (1998).

1 There are by now a few survey articles on the subject: Kakwani (1984); Foster (1984); Atkinson (1987, 1989); Seidl (1988); Chakravarty (1990), Chapts. 6 and 7; Callan and Nolan (1991), Zheng (1997). Most of the references on poverty measurement may be found in these surveys. 
and many who follow him conceptualize poverty measurement as involving two steps: the identification of the poor and the aggregation of the data on poverty into an overall index. With regard to the identification problem, the poverty-line or income method has attracted the most attention. ${ }^{2}$ This method determines a poverty-line income sufficient to attain minimum basic needs. Shortfalls of incomes from the poverty line encapsulate human deprivation. A poor person is then someone whose income falls below the poverty line. The second step is to develop an aggregation rule for the shortfalls. For reasons stated in the following section, this paper departs from the income method and explores an alternative approach which is multidimensional in nature. Unlike the income approach, human deprivation is visualized not through income as an intermediary of basic needs but in terms of shortfalls from minimum levels of basic needs themselves. The multidimensional poverty index is then an aggregation of shortfalls of all the individuals. To be more precise, let $\underline{x}_{i}, i=1,2, \ldots, n$, be the vector of $k$ basic needs of the $i$ th person and $\underline{z}=\left(z_{1}, \ldots, z_{k}\right)$ be a $k$-vector of the minimum levels of basic needs. Then a multidimensional poverty index is a real-valued function mapping $\underline{x}_{i}, i=$ $1,2, \ldots, n$, to the real line. As shown below, new issues on identification and aggregation emerge in the multi-attribute context.

This paper extends the works on poverty measurement along the following fronts:

1. This paper extends the class of sub-group consistent poverty index proposed by Foster and Shorrocks (1991) to the multidimensional context (Proposition 3) and explores the existence of a pair of relative and absolute poverty measures with the same ranking with respect to poverty (see the discussion in Sect. 8).

2. A set of axioms for multidimensional poverty measures is introduced, some of which are generalizations of those in the unidimensional context, while others are new. In particular, transfer principles for transfer-sensitive multidimensional indices are explored. We argue that direct generalizations of transfer principles from the undimensional context may not adequately encompass some types of transfers that seem intuitively to be equalizing. In this regard, a novel criterion termed "Poverty Non-decreasing Rearrangement" is introduced.

3. For the practitioners, unique classes of relative and absolute poverty measures are derived based on different sets of axioms. Some of them have their unidimensional analogues while others are new.

4. Our results demonstrate that the design of poverty measures may be very sensitive to whether the attributes may assume nonpositive values. This is particularly the case with respect to relative indices.

In Sect. 2, the rationale for multidimensional poverty measures is discussed. Section 3 puts together a set of basic axioms for poverty indices; the

2 The term "income method" is that of Sen (1981). 
concept of subgroup consistency as introduced by Foster and Shorrocks (1991) is generalized. Sections 4 and 5 look into various criteria for identifying one situation as more equal than another in a multi-attribute context. In Sects. 6 and 7, different classes of subgroup consistent poverty measures are introduced; the implications of domain restrictions are also studied. Following Foster and Shorrocks (1991), a pair of relative and absolute multidimensional poverty indices with the same inequality ordering is derived in Sect. 8. The concluding section explores future research directions. All the proofs in this paper are relegated to Appendix A.

\section{Rationale for multidimensional poverty measurement}

The concept of multidimensional poverty measurement is certainly not new. Besides the income approach, there is an alternative and more direct method. In identifying the poor, one may check whether a person meets a set of minimum basic needs. ${ }^{3}$ Each person is than characterized not just by income but by a vector of characteristics, e.g., health, housing condition, etc. ${ }^{4}$ While Sen (and many others following him) opted for the income approach (Sen 1976, 1981), he was fully cognizant that the direct method may in certain senses be superior; see, e.g., Sen (1981). Recently, there is a resurgence of interest in multidimensional measures of poverty; see, e.g., Streeten (1995), Ravallion (1996).

Is there anything to gain by adopting a multidimensional perspective? Implicit in the multidimensional approach is the view that income by itself may not be an appropriate variable on which the measurement of human deprivation is based. Nowhere is this viewpoint more prevalent than in the field of development economics. The basic needs approach, which has gradually been gaining ground among development economists and international agencies, perceives development as a sustained improvement in human basic needs and not just as income growth. ${ }^{5}$ Why is income not sufficient in reflecting other basic needs? To a certain extent, it is true that increase in purchasing power permits a poor individual to better fulfil her basic needs. However, this reasoning presupposes markets for all basic needs which do not always exist. Many basic needs assume the nature of public goods, e.g., malaria prevention in developing countries. Empirical studies on the relationship between nutri-

\footnotetext{
${ }^{3}$ See e.g., Sen (1981) and Atkinson (1989). Kanbur aptly points out that "the disputes around what the poverty line should be arise partly because the standard of living is itself a multidimensional concept." (Kanbur 1987)

${ }^{4}$ For an empirical application of the direct method, see, e.g., Atkinson (1989), Chap. 5.

5 See Streeten (1981) for an authoritative discussion of the basic needs approach to development. Among the international agencies, the World Bank devoted the 1990 World Development Report to the issue of poverty. The United Nations Development Programme (UNDP) has published annual reports on human development since 1990 (UNDP, various issues).
} 
ent intakes and income also provide evidence which further casts doubt on the close correlation between income and basic needs. ${ }^{6}$

Related to, though not equivalent to, the basic needs approach is the theoretical framework propounded by Sen $(1985,1987,1988,1992)$. In Sen's framework, what ultimately matters is what a person "manage to do or to be" and the freedom of a person to choose her functionings (Sen 1985). Attributes such as longevity, literacy, etc. and not income per se are closer approximations of these dimensions of well-being. Applying Sen's framework to poverty measurement, human deprivation may be better approximated in terms of life expectancy, literacy and so on. ${ }^{7}$

It does therefore seem surprising that the unidimensional income approach has for a long time occupied such a dominant position in poverty measurement. However, a moment of reflection suggests a few reasons to account for such a lopsided situation. Weights other than prices may at first sight seem less natural. But a second thought cannot fail but convince us that prices as weights are equally arbitrary. ${ }^{8}$ In one sense, the multidimensional approach to be expounded below is superior because it makes explicit the effects of the weighting schemes for the basic needs (or functionings) on overall poverty. Another reason for the popularity of the income approach may stem from its advantage in assigning a numerical value to the degree of poverty, while the direct approach only evaluates deprivation of each basic need one by one. ${ }^{9}$ The approach in this paper strikes a balance between the income method and the direct method by supplying a numerical representation for overall poverty while preserving the multidimensional nature of the problem. Finally, the relaxation of data on attributes other than income now renders the proposed multidimensional approach all the more possible. ${ }^{10}$

\footnotetext{
${ }^{6}$ For a survey of the literature on nutrient intakes and income growth, see Behrman and Deolalikar (1988) and Dasgupta (1993). Ravallion (1990, 1992) challenged the results of the previous studies.

7 The approach adopted in this paper falls short of the capability approach advocated by Sen $(1985,1988,1992)$. Capability measures the freedom to achieve certain important functionings. Thus the capability of a person is an opportunity set of bundles of functionings and not the functionings achieved. The multidimensional poverty indices in this paper however measure the functionings achieved. While one may agree with Sen that capability is the crux of the matter in poverty measurement, the practical implementation of the capability approach is inherently difficult because one has to have information on the opportunity sets of functionings.

8 The use of prices as weights implicitly assumes that prices are in some sense "right". Fundamentally, this position is no less value-free than using other sets of weights. Furthermore, prices may not exist for all goods when there are market failures.

9 In fact, this is one reason why Sen thought that the income approach has an edge over the direct approach (Sen 1981, p. 28).

10 The recent spate of studies akin to our multidimensional approach is a witness to the relaxation of the informational constraint, see, e.g., Ram (1982), UNDP (various issue), Maasoumi and Nickelburg (1988), Maasoumi (1989), Hirschberg et al. (1991), Slottje (1991). These studies are mostly concerned with the measurement of multidimensional welfare and inequality.
} 


\section{Basic axioms of multidimensional poverty indices}

As aptly pointed out by Ravallion (1996, p. 1333), the design of meaningful measures of multidimensional poverty calls for "a clearer axiomatic basis". This section lays down the axiomatic foundation for multidimensional poverty indices. Before introducing the axioms, an explanation of our notation is in order. $\mathbb{R}^{q}, \mathbb{R}_{+}^{q}$ and $\mathbb{R}_{++}^{q}$ are the $q$-dimensional Euclidean space and its nonnegative and positive counterparts respectively; similiarly, $\mathbb{R}_{-}^{q}$ and $\mathbb{R}_{--}^{q}$ are the $q$-dimensional negative and nonpositive Euclidean spaces respectively. $\prod_{i=1}^{k} J_{k}$ is the $k$ Cartesian product of the intervals $J_{k}$; for $J_{k}=J$ for all $k, J^{k}$ is the $k$ Cartesian product of $J{ }^{11} X^{T}$ is the transpose of the matrix $X$. For any vector $\underline{\lambda}, \operatorname{diag}(\underline{\lambda})$ is the notation for a diagonal matrix whose $j$ th diagonal element is $\lambda_{j}$. For any $k$-vectors $\underline{u}$ and $\underline{v}$, $\underline{u} \wedge \underline{v}:=\left(\min \left(u_{1}, v_{1}\right), \ldots, \min \left(u_{k}, v_{k}\right)\right), \underline{u} \vee \underline{v}:=\left(\max \left(u_{1}, v_{1}\right), \ldots, \max \left(u_{k}, v_{k}\right)\right)$. A $k$-vector of one's is denoted by $\underline{e}_{k}$ and that of zeros by $\underline{0}_{k}$. Let there be $n$ persons and $k$ attributes. Unless stated otherwise, $n \geq 3$ and $k \geq 1$. For person $i, x_{i j}$ is the amount of the $j$ th attribute accruing to the $i$ th person and $\underline{x}_{i}$ is a $k$-vector of attributes of the $i$ th person.

In the subsequent analysis, $n \times k$ matrices $X$ whose $i$ th row is $\underline{x}_{i}$, summarizing the distributions of $k$ attributes among $n$ persons, may assume two different domains with respect to the attributes. First, $x_{i j}$ may assume any value on the real line; let $\mathfrak{M}(n, k)$ be the class of $n \times k$ matrices such that $x_{i j} \in \mathbb{R}$ and $\mathfrak{M}:=\left\{X \in \bigcup_{n} \bigcup_{k} \mathfrak{M}(n, k)\right\}$. Next, for the attributes to be positive, the class of matrices is restricted to $\mathfrak{M}^{++}:=\left\{X \in \bigcup_{n} \bigcup_{k} \mathfrak{M}^{++}(n, k)\right\}$, where $\mathfrak{M i}^{++}(n, k)$ is the class of $n \times k$ matrices such that $x_{i j} \in \mathbb{R}_{++}$. Commensurate with the above notation, a vector $\underline{x}$ is an element of $\mathfrak{M}(1):=\bigcup_{k} \mathfrak{M}(1, k)$. To avoid the cumbersome exposition due to the changes in the domain restrictions, $\mathfrak{D}$ denotes either $\mathfrak{D}=\mathfrak{M}$ or $\mathfrak{D}=\mathfrak{M}^{++}$. For example, a statement with $X \in \mathfrak{D}$ means that the statement applies when $X$ is either in $\mathfrak{M}$ or in $\mathfrak{M}^{++}$; correspondingly, $\mathfrak{D}(n, k)=\mathfrak{M}(n, k) / \mathfrak{M}^{++}(n, k)$ and $\mathfrak{D}(1)=\mathfrak{M}(1) / \mathfrak{M}^{++}(1)$, etc.

With regard to the identification problem, a threshold for each attribute is determined to represent the minimum level of basic needs and $\underline{z}=$ $\left(z_{1}, \ldots, z_{k}\right)$ is a $k$-vector of thresholds such that $\underline{z} \in Z, Z \subset \bigcup_{k} \mathbb{R}_{++}^{k}$. Let $\mathfrak{P}:=$ $\left\{\underline{x} \in \mathfrak{D}(1): x_{j} \leq z_{j}\right.$, for some $\left.j\right\}$, then a person is identified as poor if her bundle $x$ is in $\mathfrak{P}$.

Definition 1. For any $X \in \mathfrak{D}$ and $\underline{z} \in Z$, the set of poor persons is defined as $\mathfrak{N}(X, \underline{z}):=\left\{i: \underline{x}_{i} \in \mathfrak{P}\right\}$.

$\Pi(X, \underline{z})$ is the corresponding number of poor persons. For example, if $k=2, \underline{z}=(2,3)$ and $\underline{x}_{i}=(1,5)$, then this individual is still considered poor even though the value of the second attribute exceeds 3 . To simplify the exposition below, $X(\underline{z})$ is defined as a $\Pi(X, \underline{z}) \times k$ matrix derived from $X$ by selecting $\underline{x}_{i}$ such that $i \in \mathfrak{N}(X, \underline{z})$. Increases in those attributes above

${ }^{11}$ For example, $(-\infty, 1]^{q}$ is the $q$-product of the interval $(-\infty, 1]$. 
their poverty thresholds do not eventually lift a person out of poverty, so long as there is at least one attribute falling beneath the threshold. This formulation, in a sense, emphasizes the essentiality of each attribute. The above definition raises some difficult ethical issues. In so far as each attribute is an essential component of well-being, is a very rich person still poor if she fails to attain the thresholds of all the other basic needs? There does not seem to be an obvious answer. In the final analysis, how reasonable the identification rule is depends, inter alia, on the attributes included and how imperative these attributes are to leading a meaningful life. If the attributes are, say, life expectancy (or other health indicators) and income, it seems reasonable to argue that a person is still poor if her life expectancy falls below the threshold, even though her real income is quite high. ${ }^{12} \mathrm{Or}$, a penniless person is still poor even if she enjoys a long life. ${ }^{13}$ The povertyline approach may be interpreted as adopting implicitly the opposite viewpoint. In so far as all the attributes may be assigned some shadow prices, a person $m$ is poor if $m \in\left\{i: \sum_{j} x_{i j} q_{i} \leq \sum_{j} z_{j} q_{j}\right\}$ where $\underline{q}$ is a vector of shadow prices or welfare weights. The attributes are thus perfectly substitutable.

A multidimensional poverty index is a function mapping some $n \times k$ matrix $X$ and $\underline{z} \in Z$ to the real line, i.e., $P: \mathfrak{D} \times Z \rightarrow \mathbb{R}$ (recall that $\mathfrak{D}$ may be either $\mathfrak{M}$ or $\mathfrak{M}^{++}$depending on the context of analysis). The index is assumed to satisfy the following basic axioms throughout the paper:

Symmetry (SM). $P(X ; \underline{z})=P(\Pi X ; \underline{z})$, where $\Pi$ is an $n \times n$ permutation matrix.

Replication Invariance (RI). $P(X ; \underline{z})=P\left(X^{r} ; \underline{z}\right)$ where $X^{r}$ is an $r$-time replication of $X$.

Monotonicity (MN). $P(X ; \underline{z}) \leq P(Y ; \underline{z})$ whenever $X$ is derived from $Y$ by increasing any one attribute with respect to which a person is poor.

Focus (FC). The poverty index remains unchanged if any attribute such that $x_{i j} \geq z_{j}$ is increased.

12 As pointed out by a referee, it is not obvious how one can identify a threshold for life expectancy. I do not have a good answer how one should choose the threshold. Perhaps a conceivable approach is to infer the life expectancy implied by an adequate level of nutrient intake as suggested by the medical profession. Though such an approach is not likely to be free of controversy.

${ }^{13}$ The definition of the poor in the text is not the only one conceivable. As kindly suggested by a referee, one may define a person as poor if and only if all the attributes simultaneously fall below the respective thresholds. This formulation may not be entirely free of controversy as explained in the text. My hesitation to adopt such a defintion is shared by Bourguignon and Chakravarty (1997a) whose paper contains an interesting and useful discussion of the issue. They give an interesting example of an old beggar. In so far as income and longevity are two attributes, our definition for poverty classifies an old beggar as poor in so far as his income falls below the respective threshold even though she may enjoy a long life. However, the less restrictive definition suggested by the referee classifies the old beggar as nonpoor, though such a classification is not entirely obvious. 
Continuity (CN). For any $\underline{z}, P$ is a continuous function of $X \in \mathfrak{D} .{ }^{14}$

These are generalizations of those basic axioms in Foster and Shorrocks (1991) and many other studies on unidimensional poverty measures. By the symmetry axiom, permuting the bundles of the basic needs among the $n$ persons does not change the poverty level. Replication invariance ensures that poverty depends on the distributions of the basic needs and their shortfalls below $\underline{z}$ rather than on the size of the population. Basic needs distributions corresponding to different population sizes may then be compared. MN ensures that the poverty index does not increase when the conditions of the poor improve.

FC is somewhat different from that in the unidimensional context and an explanation is thus warranted. In so far as the $j$ th attribute of a poor person exceeds $z_{j}$, giving more of that attribute to her does not affect the level of poverty even though that person remains poor. ${ }^{15}$ The present axiom is reduced to that in the unidimensional setting. In conjunction with $\mathrm{MN}$, for $x_{i} \geq z_{i}$, an increase in $x_{i}$ of a poor person does not increase poverty. Finally, continuity of $P$ precludes abrupt changes in $P$ for small changes in $X$.

In the subsequent discussion, we define a multidimensional poverty measure as follows:

Definition 2. A multidimensional poverty measure is a real-valued function $P: \mathfrak{D} \times Z \rightarrow \mathbb{R}$ satisfying $S M, R I, M N, F C$ and $C N$.

Furthermore, in the subsequent analysis, we are only interested in those classes of multidimensional poverty measures which are not identically equal to a constant as defined below:

Definition 3. A multidimensional poverty measures is nontrivial if it does not always assume a constant value for different $X$.

It is desirable to have a poverty index which does not increase if the degree of poverty of a subgroup of population decreases. Foster and Shorrocks (1991) exhaustively characterized this class of poverty indices for the povertyline approach. Subgroup consistency may easily be extended to the multidimensional setting:

Subgroup Consistency (SC). For any $n$ and $k$ such that $X_{1}$ and $Y_{1}$ are $n \times k$ matrices and $X_{2}$ and $Y_{2}$ are $m \times k$ matrices, with $X^{T}:=\left[X_{1}^{T}, X_{2}^{T}\right]$ and $Y^{T}:=$ $\left[Y_{1}^{T}, Y_{2}^{T}\right], P(X ; \underline{z})>P(Y ; \underline{z})$ whenever

$$
P\left(X_{1} ; \underline{z}\right)>P\left(Y_{1} ; \underline{z}\right) \text { and } P\left(X_{2} ; \underline{z}\right)=P\left(Y_{2} ; \underline{z}\right) \text {. }
$$

${ }^{14}$ Foster and Shorrocks (1991) also explored the case when continuity is replaced by restricted continuity, i.e., $P(X ; \underline{z})$ is continuous on the restricted domain whereby $x_{i j} \leq z_{j}$.

${ }^{15}$ FC implicitly assumes that there is in a sense no substitution between attributes, i.e., increasing those attributes that are above their thresholds cannot compensate for the shortfalls of other attributes and pull a person out of poverty. This axiom is thus commensurate with definition 1 above regarding the identification of poverty. 
One can easily adapt the results in Foster and Shorrocks (1991) to show that any subgroup consistent multidimensional poverty measure must have the form as in Eq. (1).

Proposition 1. A multidimensional poverty index $P(X ; z)$ satisfies $S C$ if and only if:

$$
P(X ; \underline{z})=G\left[\frac{1}{n} \sum_{i=1}^{n} \phi\left(\underline{x}_{i}, \underline{z}\right) ; \underline{z}\right]
$$

where $G$ is a strictly increasing and continuous function; $\phi: \mathfrak{D}(1) \times Z \rightarrow \mathbb{R}$ is continuous and non-increasing with respect to the attributes; $\phi(\underline{x} \wedge \underline{z}, \underline{z})=$ $\phi(\underline{x}, \underline{z})$; furthermore, $\phi(\underline{x}, \underline{z})=0$ when $x_{i} \geq z_{i}$ for all $i=1,2, \ldots, k$.

All the proofs in this paper are relegated to the appendix. Those who are interested in comparing our results to those in Foster and Shorrocks (1991) may also consult Lemma 1 in Sect. 7 and Propositions 7 and 8 in Sect. 8 below. In the subsequent analysis, other axioms are introduced sometimes restricting Eq. (1) to specific classes of poverty measures.

\section{Distribution-sensitive poverty indices}

In his well-known critique of the poverty-gap measure and the headcount ratio, Sen (1976) suggested that a poverty index should be sensitive to inequality among the poor. If income inequality among the poor decreases, the poverty index should fall. Well-known criteria for ordering income vectors in the literature on inequality measurement are often invoked for designing transfer-sensitive poverty measures. One such criterion is the Pigou-Dalton principle whereby a mean-preserving transfer of income from the rich to the poor reduces the index. Equivalently, $y=B \underline{x}$, where $B$ belongs to $\mathfrak{B}$, the set of bistochastic but not permutation matrices, if and only if $y$ may be derived from $\underline{x}$ via a sequence of Pigou-Dalton transfers; see, e.g., Dasgupta et al. (1973). ${ }^{16}$ The different criteria are thus different characterizations of the same inequality ordering. In the multidimensional context, the two criteria may be generalized as follows: ${ }^{17}$

(a) Uniform Pigou Dalton transfer (UPD): For any $n$, let any $n \times n$ matrix $T$ be referred to as a Pigou-Dalton transfer matrix whenever $T=\lambda I+$ $(1-\lambda) Q, 0<\lambda<1, I$ is an $n \times n$ identity matrix and $Q$ is an $n \times n$ permutation matrix interchanging two coordinates of any vector (see Marshall and Olkin 1979, p. 21); let $\mathfrak{I}:=\bigcup_{n} \mathfrak{I}(n)$, where $\mathfrak{I}(n)$ is the class of $n \times n$ PigouDalton transfer matrix, then $(Y, X) \in \mathrm{UPD}, X, Y \in \mathfrak{D}$, if and only if $Y=T X$, where $T$ is a product of matrices in $\mathfrak{I}$.

16 A square matrix $B=\left[b_{i j}\right]$ is bistochastic if and only if $\Sigma_{i} b_{i j}=1$ for each $j$ and $\sum_{j} b_{i j}=1$ for each $i$.

17 See, e.g., Tsui (1999) for details. 
(b) Uniform Majorization (UM): $(Y, X) \in \mathrm{UM}$ if and only if $Y=B X$, $B \in \mathfrak{B}$.

It is obvious that if $(Y, X) \in \mathrm{UPD}$, then $(Y, X) \in \mathrm{UM}$. The converse is however not true; see, e.g., Kolm (1977); Mosler (1994, 1995); Joe and Verducci (1992); Koshevoy (1995); Koshevoy and Mosler (1996) and Tsui (1999). As show below, these two concepts are, however, equivalent in the context when poverty measures are subgroup consistent.

In the unidimensional context, numerous transfer axioms are conceivable; see, e.g., Donaldson and Weymark (1986). To control the length of our discussion, we shall limit our attention to redistribution among the poor. When $k=1$, a transfer among the poor with respect to either UPD or UM does not change the set of poor persons. This is no longer true when $k>1$ due to our definition of poverty. It is obvious that a transfer with respect to either UPD or UM may lead to a decrease in the number of poor persons. ${ }^{18}$ It therefore seems reasonable to require that the poverty index falls as a result. To capture this idea, we may entertain the following axiom: ${ }^{19}$

Poverty Decreasing Minimal Transfer Axiom (PDMT) with respect to a majorization criterion. $P(Y ; z)<P(X ; \underline{z})$ if the transfer is among the poor.

PDMT reduces to the minimal transfer of Donaldson and Weymark (1986) when $k=1$. To ease exposition, we shall refer to PDMT with respect to the $m$ th majorization criterion, $m=\mathrm{UPD}, \mathrm{UM}$, as $\operatorname{PDMT}(m)$. PDMT is however not consistent with FC. To see this, let the vectors of attributes of two persons be $(1,3)$ and $(1,5)$ and the vector of poverty thresholds be $(2,2)$. The two are thus classified as poor. If there is an equalizing transfer (with respect to either of the above criteria) such that both of them have $(1,4)$, should the level of poverty fall? Since the equalization pertains to the second attribute whereby both of them have amounts exceeding the poverty threshold, it is arguable whether there is a decrease in deprivation even though there is a decrease in inequality among the poor. In general, we have the following impossibility result:

Proposition 2. There does not exist any subgroup consistent multidimensional poverty index satisfying PDMT(UM) or PDMT(UPD).

One way to get around the above impossibility result is to replace PDMT by the following axiom:

Poverty Non-increasing Minimal Transfer Axiom (PNMT) with respect a majorization criterion. $P(Y ; \underline{z}) \leq P(X ; \underline{z})$ if transfers are among the poor.

${ }^{18}$ Let there be two attributes and the vector of poverty thresholds be $(2,2)$; furthermore, the vectors for two persons are $(5,1)$ and $(1,5)$. By definition, they are poor. If a equalizing transfer results in both of them having $(3,3)$, then they become nonpoor.

${ }_{19}$ Another possibility is to restrict the transfers to the domain whereby $x_{i j} \leq z_{j}$ for all $j \in\{1,2, \ldots, k\}$. In this case, poverty may fall without violating the other axioms. 
We shall adhere to PNMT in the subsequent discussion. Again, PNMT(UM) and PNMT(UPD) corresponds to PNMT under different majorizations criteria. It is easy to show that the function $\phi($.$) in Eq. (1) is restricted to be$ convex by PNMT.

Proposition 3. The class of subgroup consistent multidimensional poverty indices as depicted by Eq. (1) satisfies PNMT(UM) or PNMT(UPD) if and only if $\phi$ is convex.

A similar result in the context of multidimensional inequality may be found in Kolm (1977).

\section{Poverty-nondecreasing rearrangement}

This section introduces a type of transfer which is peculiar to multidimensional poverty indices. Similar conditions were first introduced by Atkinson and Bourguignon (1982) and later by Dardanoni (1995) and Tsui (1999) into the literature on multidimensional inequality measurement. PNMT in some sense ensures that a poverty index is sensitive to the dispersion of the attributes among the poor. It is however not difficult to imagine situations which are compellingly inequitable and yet not covered by $\operatorname{PNMT}(m)$. Let us consider a situation with three poor persons and three attributes whose poverty thresholds are, say, $z=(4,4,4)$. The initial distribution of the attributes is summarized by the matrix $X_{0}$ below, where each row of $X_{0}$ is the vector of attributes of the respective individual. Suppose that $X_{0}$ is subject to the following sequence of resource redistributions:

$$
X_{0}=\left(\begin{array}{lll}
1 & 2 & 3 \\
2 & 3 & 2 \\
3 & 1 & 1
\end{array}\right) \rightarrow X_{1}=\left(\begin{array}{lll}
1 & 1 & 1 \\
2 & 3 & 2 \\
3 & 2 & 3
\end{array}\right) \rightarrow X_{2}=\left(\begin{array}{lll}
1 & 1 & 1 \\
2 & 2 & 2 \\
3 & 3 & 3
\end{array}\right)
$$

From $X_{0}$ to $X_{1}$, the resources are exchanged between the first and the third person such that the third person gets more of everything. Likewise, the transformation from $X_{1}$ to $X_{2}$ is such that the third person has more of every thing after the transformation. In each step of the sequence, there is no change in the number of poor persons and there is an increase in the correlation of the attributes, i.e., a person who has more of one attribute has more of the other attributes. Roughly speaking, the richer poor are getting even better after each rearrangement. Intuitively, poverty should increase or at least be nondecreasing as a result of these transformations. In the example above, the number of poor persons remain unchanged. However, if $\underline{z}$ is instead equal to $(2.5,2.5,2.5)$, the third person crosses the poverty thresholds as a result of the transformation from $X_{1}$ to $X_{2}$. It is thus ambiguous whether poverty should increase in this case.

In general we may introduce an axiom which captures the above ideas by 
resorting to some concepts in Borland and Proschan (1988). Let $\mathfrak{X}(p, q)=$ $\left\{X \in \mathfrak{D}(p, q): x_{11} \leq x_{21} \leq \cdots \leq x_{p 1}\right\}$ (i.e., the elements in the first column are arranged in increasing order) and $\mathfrak{X}=\bigcup_{p} \bigcup_{q} \mathfrak{X}(p, q)$. A new type of transfers is introduced:

Definition 4. For any $p$ and $q$ such that the matrix $X \in \mathfrak{X}(p, q)$, a transfer with respect to two rows, $i$ and $j, i<j$, is basic arrangement-increasing if and only if the post-transfer vectors become: $\underline{y}_{m}:=\underline{x}_{m}, m \notin\{i, j\}, \underline{y}_{i}:=\underline{x}_{i} \wedge \underline{x}_{j}$ and $\underline{y}_{j}:=\underline{x}_{i} \vee \underline{x}_{j}$.

For example, in the numerical example above, $X_{1}$ is derived from $X_{0}$ by a basic arrangement-increasing transfer. The next axiom ensures that the poverty index is non-decreasing (i.e., it may remain unchanged) with respect to a basic arrangement-increasing transfers:

Poverty-Nondecreasing Rearrangement (PNR). let $X \in \mathfrak{D}$. Suppose that $Y$ is derived from $X$ by a finite sequence of basic-rearrangement increasing transfers among the poor with no one becoming nonpoor due to the transfers. Then $P(X ; \underline{z}) \leq P(Y ; \underline{z})$.

To restrict the transfers to the case when no one becomes nonpoor is to avoid those ambiguous situations whereby someone becomes nonpoor while the gaps among some persons become larger. Another formulation is for the poverty index to strictly increase with respect to a poverty basic arrangementincreasing transfers. If this formulation is adopted, some classes of poverty measures derived below may be eliminated.

Next, to elucidate the restriction of PNR on the class of subgroupconsistent multidimensional poverty measure, let us introduce the class of L-superadditive functions; see Marshall and Olkin (1979, pp. 150-155). A function $f$ is L-superadditive if and only if

$$
f(\underline{x})+f(\underline{y}) \leq f(\underline{x} \wedge \underline{y})+f(\underline{x} \vee \underline{y}), \quad \underline{x}, \underline{y}, \underline{x} \wedge \underline{y}, \underline{x} \vee \underline{y} \in E \subseteq \mathbb{R}^{k} .
$$

If $f($.$) is differentiable, (2) is equivalent to the following condition:$

$$
f_{i j}:=\frac{\partial f(\underline{u})}{\partial u_{i} \partial u_{j}} \geq 0 \quad i \neq j, \underline{u} \in E .
$$

The next proposition identifies the restriction on the class of $\phi$ in Eq. (1) imposed by PNR. ${ }^{20}$

20 The fact that $\phi(., \underline{z})$ is convex as required by distribution sensitivity does not guarantee L-subadditivity. To see this, let $k=2$, then $\phi$ is convex if and only if $\phi_{11} \geq 0$, $\phi_{22} \geq 0$ and $\phi_{11} \phi_{22}-\phi_{12}^{2} \geq 0$ where $\phi_{i j}$ is the partial derivative of $\phi$ with respect to the $i$ th and $j$ th argument. Convexity of $\phi$ does not impose any restriction on $\phi_{12}$. However, L-subadditivity requires that $\phi_{12} \geq 0$. 
Proposition 4. A subgroup consistent multidimensional poverty measure satisfies $P N R$ if and only if $\phi$ in Eq. (1) is L-superadditive on $\mathfrak{P}$.

If $\phi$ is differentiable, $\partial \phi\left(\underline{x}_{i}\right) / \partial x_{i \ell} \partial x_{i m} \geq 0 .{ }^{21}$

\section{Ratio-scale invariance and specific classes of relative poverty measures}

In the literature on undimensional poverty indices, scale invariance properties are often invoked, see, e.g., surveys by Foster (1984); Seidl (1988) and Chakravarty (1990); Zheng (1997). Similar properties may be considered in the multidimensional context. A poverty index may be defined as relative if it satisfies the following scale-invariance axiom:

Ratio-Scale Invariance (RS). For any $X \in \mathbb{D}$ and $\underline{z} \in Z, P(X \Lambda ; \underline{z} \Lambda)=P(X ; \underline{z})$ where $\Lambda:=\operatorname{diag}(\underline{\lambda})$ and $\underline{\lambda} \in \bigcup_{k} \mathbb{R}_{++}^{k}$.

In the unidimensional context, $\mathrm{RS}$ is often justified on the ground that poverty measures should not be sensitive to a change in the unit of measurement. A similar interpretation may be invoked in the multidimensional setting.

There are many functional forms compatible with the axioms introduced so far. Next, we introduce an axiom that, in conjunction with other axioms, reduces $P$ to a unique class of multidimensional poverty measures. Let $P(X ; \underline{z})$ $\leq P(Y ; \underline{z})$; suppose that there is an adjustment of the vector of poverty thresholds upward from $\underline{z}$ to $\underline{z}^{\prime}$ such that $z_{j} \leq z_{j}^{\prime}$. If the new poverty thresholds identify the same group of people as poor, then the ordinal ranking of $X$ and $Y$ should remain unchanged under $\underline{z}^{\prime}$. The above scenario is captured by the following axiom:

Poverty Criteria Invariance (PCI). Let $\underline{z}, \underline{z}^{\prime}$ be such that $z_{j} \neq z_{j}^{\prime}$; then

$$
P(X ; \underline{z}) \leq P(Y ; \underline{z}) \Leftrightarrow P\left(X ; \underline{z}^{\prime}\right) \leq P\left(Y ; \underline{z}^{\prime}\right) .
$$

whenever $X(\underline{z})=X\left(\underline{z}^{\prime}\right)$ and $Y(\underline{z})=Y\left(\underline{z}^{\prime}\right)$.

Since there is often no consensus on $\underline{z}$, PCI ensures that there is no dramatic change in the evaluation of poverty for changes in $z$ not affecting the number of the poor. Combining with RS, PCI leads inexorably to unique classes of

${ }^{21}$ One referee comments that the idea behind poverty-increasing (non-decreasing) rearrangement implicitly assumes that the attributes are substitutable. If substitutability means that reducing the amount of one attribute from a poor person may be compensated by a increase in another attribute such that her degree of poverty remains unchanged, then poverty-increasing (non-decreasing) rearrangement is not exactly implied by that definition of substitutability. What the axiom really says is that the poverty-reducing impact of giving more of an attribute to a poor person decreases as the person in question has larger endowments of other attributes. It follows that taking away a bit of an attribute from one poor person A to another poor person B with more of other attributes than $\mathrm{A}$ has the net effect of increasing poverty. This is the implication of $\partial \phi\left(\underline{x}_{i}\right) / \partial x_{i \ell} \partial x_{i m} \geq 0$ where the function $\phi\left(\underline{x}_{i}\right)$ essentially captures the impact of $\underline{x}_{i}$ on the $i$ th person. 
poverty measures that are multidimensional generalizations of their oneattribute counterparts. One drawback of PCI is that it rules out the possibility of changes in shortfalls, i.e., from $z-x_{i k}$ to $z^{\prime}-x_{i k}$, leading to reversals of ordinal rankings. In so far as differential ethical weights are assigned to shortfalls of attributes, it is not inconceivable that a change in $\underline{z}$ may reverse the ordinal ranking of $X$ and $Y$. Indeed, a multidimensional generalization of the class of Foster-Greer-Thorbecke poverty measures is not consistent with PCI.

Domain restrictions turn out to have a rather dramatic impact on the design of relative poverty measures. In the rest of this section, two cases are explored.

Case $A . X \in \mathfrak{M}^{++}$and $\underline{z} \in \mathbb{R}_{++}^{k}$. In the unidimensional context, it is common to restrict income to be positive. In conjunction with PCI and the other axioms, the only class of subgroup consistent multidimensional poverty measures turns out to be a generalization of the class of unidimensional measures proposed by Chakravarty (1983).

Proposition 5. Let $X \in \mathfrak{M}^{++}$and $\underline{z} \in \mathbb{R}_{++}^{k} \cdot A$ nontrivial multidimensional poverty index satisfies $S C, P N M T(U M)$ (or $P N M T(U P D)$ ), $P N R, R S, P C I$ if and only if there exists a strictly increasing transformation of $P(X ; z)$ given by

$$
P_{1}(X ; \underline{z})=\frac{1}{n} \sum_{i=1}^{n}\left[\prod_{j=1}^{k}\left(\frac{z_{j}}{x_{i j} \wedge z_{j}}\right)^{\alpha_{j}}-1\right]
$$

$\alpha_{i} \geq 0, i=1,2, \ldots, k$, and the parameters $\alpha_{i}$ has to be chosen such that the function $\phi^{*}(\underline{\theta})=\prod_{i=1}^{k} \theta_{i}^{-\alpha_{i}}, \theta_{i} \in(0,1]$ is convex with respect to $\left(\theta_{1}, \ldots, \theta_{k}\right)$; or

$$
P_{2}(X ; \underline{z})=\frac{1}{n} \sum_{i=1}^{n} \sum_{j=1}^{k} \delta_{j} \ell n\left(\frac{z_{j}}{x_{i j} \wedge z_{j}}\right)
$$

where $\delta_{i} \geq 0, i=1,2, \ldots, k$.

For $P_{1}$, let us clarify the restrictions on $\alpha_{i}$ for the case when $k=2$. By $\mathrm{MN}, \alpha_{i} \geq 0$. For the function $\phi^{*}(\underline{\theta})$ to be convex, $\alpha_{1}\left(\alpha_{1}+1\right) \geq 0$ and $\alpha_{1} \alpha_{2}\left(1+\alpha_{1} \alpha_{2}\right) \geq 0$. So long as $\alpha_{i} \geq 0$, all the restrictions are obviously satisfied. It is also clear that $P_{1}$ satisfied PNR because $\alpha_{1} \alpha_{2} \geq 0$. Next, It is to be noted that $P_{2}$ satisfies PNR in the sense that the value of the expression remains unchanged with respect to a poverty-reducing re-arranagement, i.e., $P_{2}(Y ; \underline{z})=P_{2}(X ; \underline{z})$ whenever $Y$ is derived from $X$ via a poverty-reducing re-arranagement. If poverty has to increase with respect to PNR, then $P_{2}(Y ; \underline{z})$ will be eliminated. Finally, $P_{2}(Y ; \underline{z})$ is a multidimensional generalization of the Watts poverty index. ${ }^{22}$

Case $B . \mathfrak{D}=\mathfrak{M}$ and $\underline{z} \in \mathbb{R}_{++}^{k}$. If $x_{i j}$ can assume non-positive values, the resulting class of poverty measures is completely different from Eq. (3). Zheng (1997) alluded to the problem encountered by relative poverty measures in the

22 For an axiomatic characterization of Watt's index in the unidimensional context, see Zheng (1993). 
unidimensional context when income is negative. It is not uncommon to have survey households with negative incomes. In so far as income may be one of the attributes in $X$, it seems interesting to find out what effect negative incomes has on relative multidimensional poverty measures. The next proposition demonstrates that a very different class of measures emerge when the attributes may assume any value on the real line.

Proposition 6. Let $\underline{z} \in \mathbb{R}_{++}^{k}$ and $\mathfrak{D}=\mathfrak{M}$. A nontrivial multidimensional poverty index satisfies SC, PNMT(UM) (or PNMT(UPD)), PNR, RS, PCI if and only if there is at most one attribute, say, the $m^{\text {th }}$ attribute, and an increasing transformation of $P$ given by

$$
P_{3}(X ; \underline{z})=\left\{\begin{array}{l}
1-\frac{1}{n} \sum_{i=1}^{n}\left(\frac{x_{i m} \wedge z_{m}}{z_{m}}\right)^{r_{m}}, \quad x_{i m}>0 \\
1-\frac{a}{n} \sum_{i=1}^{n}\left|\frac{x_{i m}}{z_{m}}\right|^{r_{m}}, \quad a \leq-1, x_{i m} \leq 0,
\end{array}\right.
$$

for some $m \in\{1,2, \ldots, k\}$ and $r_{m}>0$.

In the multidimensional setting when attributes may assume nonpositive values, Proposition 6 effectively precludes mutidimensional analysis of poverty since the resulting class of poverty measures can at most be sensitive to one attribute. Furthermore, the index is not sensitive to any sequence of basic arrangement-increasing transfers. Zheng (1997) contends that RS may be "nonsensical" if the attribute in question can be negative. The problem with respect to RS seems to be aggravated in the multidimensional context.

\section{Translation-scale invariance and a specific class of absolute poverty indices}

A unidimensional poverty index is absolute if and only if adding a constant to the income of each person and the poverty-line income does not change the degree of poverty. In the multidimensional context, the following axiom is a natural extension:

Translation-Scale Invariance (TS). $P(X+\Gamma ; \underline{z}+\underline{t})=P(X ; \underline{z})$, where $X$, $X+\Gamma \in \mathfrak{D}, \underline{z}+\underline{t} \in Z$ and $\Gamma$ belongs to the class of matrices $\mathfrak{I} \subset \mathfrak{M}\left(\right.$ or $\left.\mathfrak{M}^{+}\right)$ with identical rows $\underline{t}:=\left(t_{1}, \ldots, t_{k}\right)$.

TS is popularized by Kolm $(1976 a, b)$ in the context of inequality measurement. Other than Blackorby and Donaldson (1980) who suggested specific classes of absolute poverty measures, absolute indices are less popular than their relative counterparts as tools for empirical studies. In the rest of this section, different domain restrictions with respect to $\mathfrak{D}, Z$ and $\mathfrak{J}$ are explored.

Before identifying the class of poverty measures compatible with TS, the following lemma, a straightforward generalization of a similar result in Foster and Shorrocks (1991), is useful to our subsequent analysis. 
Lemma 1. For (a) $\mathfrak{D}=\mathfrak{M}^{++}, \underline{z} \in \mathbb{R}_{++}^{k}$ and $\mathfrak{I} \subset \mathfrak{M}^{+}$, (b) $\mathfrak{D}=\mathfrak{M}, \underline{z} \in \mathbb{R}_{++}^{k}$, $\mathfrak{J} \subset \mathfrak{M}^{+}$and and $(c) \mathfrak{D}=\mathfrak{M}, \underline{z} \in \mathbb{R}^{k}, \mathfrak{I} \subset \mathfrak{M}$, a subgroup consistent multidimensional poverty index satisfies translation-scale invariance if and only if

$$
P(X ; \underline{z})=G_{A}\left[\frac{1}{n} \sum_{i=1}^{n} \psi\left(\underline{z}-\underline{x}_{i}\right) ; \underline{z}\right]
$$

where $G_{A}$ is a strictly increasing function, $\psi\left(\underline{z}-\underline{x}_{i}\right)=\psi\left(\underline{z}-\underline{x}_{i} \wedge \underline{z}\right)$, and $\psi\left(\underline{z}-\underline{x}_{i}\right)=0$ when $x_{i j} \geq z_{j}$ for all $j$.

The next proposition derives a unique class of mulitidimensional absolute poverty measures.

Proposition 7. For (a) $\mathfrak{D}=\mathfrak{M}^{++}, \underline{z} \in \mathbb{R}_{++}^{k}$ and $\mathfrak{J} \subset \mathfrak{M}^{+}$, (b) $\mathfrak{D}=\mathfrak{M}$, $\underline{z} \in \mathbb{R}_{++}^{k}, \mathfrak{J} \subset \mathfrak{M}^{+}$and $(c) \mathfrak{D}=\mathfrak{M}, \underline{z} \in \mathbb{R}^{k}, \mathfrak{J} \subset \mathfrak{M}$, the class of nontrivial multidimensional poverty indices satisfies SC, PCI, PNMT(UPD) (or $P N M T(U M)), P N R$ and TS if and only if there exists some strictly increasing function of $P$ given by

$$
P_{4}(X ; \underline{z})=\frac{1}{n} \sum_{i=1}^{n}\left(\prod_{j=1}^{k} \exp \left\{r_{j}\left(z_{j}-x_{i j} \wedge z_{j}\right)\right\}-1\right), \quad r_{j} \geq 0
$$

or

$$
P_{5}(X ; \underline{z})=\frac{1}{n} \sum_{i=1}^{n} \sum_{j=1}^{k} c_{j}\left(z_{j}-x_{i j} \wedge z_{j}\right), \quad c_{j} \geq 0
$$

with at least one $r_{j}$ and $c_{j}$ strictly positive. Furthermore, the parameters $r_{j}$ are restricted in such a way that the function $\prod_{i=1}^{k} \exp \left\{r_{j}\left(z_{j}-x_{j}\right)\right\}$ is convex.

It is to be noted that $P_{5}$ will be eliminated if a strict version of PNR is adopted.

\section{Scale compatibility}

In the unidimensional context, Foster and Shorrocks (1991) ask whether there exists a pair of relative and absolute indices which are compatible in the sense that they have the same ordering for any two income vectors $\underline{x}$ and $y$. They have shown that the resulting class of relative indicecs is the FosterGreer-Thorbecke (FGT) measure. It is tempting to explore whether such a pair of indices exists in the multidimensional context. The axiom of Foster and Shorrocks may be reformulated as follows:

Scale Compatibility (SCM). Let $P(X ; \underline{z})$ be a relative index and $P^{\prime}(X ; \underline{z})$ an absolute index. Then, $P(X ; \underline{z}) \geq P(Y ; \underline{z}) \Leftrightarrow P^{\prime}(X ; \underline{z}) \geq P^{\prime}(Y ; \underline{z}), X, Y \in \mathfrak{D}$, $z \in Z$.

We only focus on the case when $\mathfrak{D}=\mathfrak{M}^{++}, \underline{z} \in \mathbb{R}_{++}^{k}$ and $\mathfrak{I} \subset \mathfrak{M}^{+}$since not much is added to our analysis for other domain restrictions. It turns out that SCM is incompatible with other axioms. 
Proposition 8. If $\mathfrak{D}=\mathfrak{M}^{++}, \underline{z} \in \mathbb{R}_{++}^{k}$ and $\mathfrak{I} \subset \mathfrak{M}^{+}$, there does not exist a nontrivial multidimensional poverty index simultaneiously satisfying SCM, PNMT(UM) (or PNMT(UPD)) and PNR.

The details of the proof is relegated to the Appendix. To see why such an impossibility result is obtained, it is illuminating to look into the case when $k=2$. Owing to SCM, $\phi^{*}\left(x_{1} / z_{1}, x_{2} / z_{2}\right)=\eta\left(1-x_{1} / z_{1}\right)^{\alpha_{1}}\left(1-x_{2} / z_{2}\right)^{\alpha_{2}}$ for the class of relative indices (see the proof in the Appendix). Nontriviality and $\mathrm{MN}$ implies that $\eta$ is positive, while $\alpha_{1}$ and $\alpha_{2}$ are nonnegative with at least one of them positive. Since $\phi^{*}$ is convex, its Hessian matrix is positive semidefinite, implying that $\phi_{i i}^{*}$ is nonnegative and thus $\alpha_{i} \geq 1$. Furthermore, $\phi_{11}^{*} \phi_{22}^{*}-$ $\phi_{12}^{* 2} \geq 0$, so that $1-\alpha_{1}-\alpha_{2} \geq 0$. The last restriction cannot, however, be satisfied as $\alpha_{i} \geq 1$.

\section{Conclusion}

This paper is one of the first attempts to tackle the problem of multidimensional poverty. Hopefully, the paper clarifies the axiomatic basis for the design of multidimensional poverty measures. To control the length of this paper, I have avoided introducing variations of the axioms introduced. For example, there are certainly other variants of transfer axioms that one can entertain. One research direction not followed in this paper is the ethical approach propounded by Blackorby and Donaldson (1980). Many of the concepts and axioms developed in this paper are obviously useful in the design of ethical poverty measures. A detailed discussion of ethical multidimensional poverty indices is however beyond the scope of this paper.

\section{Appendix A}

Proof of Proposition 1. The proof is a straightforward extension of that in Foster and Shorrocks (1991) and is thus not included. It is available on request.

Proof of Proposition 2. Let us first show that the statement is true with respect to PMT(UPD). Suppose that the $\ell$ th and $m$ th persons are poor; furthermore, $x_{\ell s}>z_{s}, x_{m s}>z_{s}$, while $x_{\ell t}=x_{m t}<z_{t}$, for all $t \neq s$. Let $Y$ be another matrix derived from $X$ by a uniform Pigou-Dalton transfer between $\ell$ and $m$; $\mathrm{PMT}(\mathrm{UPD})$ requires that $P(Y ; \underline{z})<P(X ; \underline{z})$. Since $y_{\ell s}, y_{m s}>z_{s}$ and $y_{\ell t}=$ $y_{m t}<z_{t}$ for all $t \neq s, \phi\left(y_{i}, \underline{z}\right)=\phi\left(\underline{x}_{i}, \underline{z}\right)$ by $\mathrm{FC}$ and thus $P(Y ; \underline{z})=P(X ; \underline{z})$, contradicting PMT(UPD). The same is true with respect to PMT(UM) because UPD implies majorization with respect UM.

Q.E.D.

Proof of Proposition 3. The proof is straightforward and is thus not included. It is available on request. A similar proof may be found in Kolm (1977).

Proof of Proposition 4. The proof is straightforward and is thus not included. It is available on request. 
Proof of Proposition 5. Let us first restrict the domain of $P$ to $\mathfrak{M}^{++}(\underline{z}) \times \mathbb{R}_{++}^{k}$, where $\mathfrak{M}^{++}(\underline{z}):=\left\{X \in \mathfrak{M}^{++}: \underline{x}_{i} \leq \underline{z}\right.$ for all $\left.i\right\}$, and $P(X ; \underline{z}) \leq P(Y ; \underline{z})$. For any $\underline{z}^{1} \geq \underline{z}$ such that $X(\underline{z})=X\left(\underline{z}^{1}\right)$ and $Y(\underline{z})=Y\left(\underline{z}^{1}\right), P\left(X ; \underline{z}^{1}\right) \leq P\left(Y ; \underline{z}^{1}\right)$ by PCI. Then, by RS:

$$
P\left(X^{1} ; \underline{\tau}\right) \leq P\left(Y^{1} ; \underline{\tau}\right) \Leftrightarrow P\left(X^{1} ; \underline{e}_{k}\right) \leq P\left(Y^{1} ; \underline{e}_{k}\right),
$$

where the $j$ th columns of $X^{1}$ and $Y^{1}$ are equal to the corresponding columns of $X$ and $Y$ divided by $z_{j}^{1} ; \tau_{j}$, the $j$ th element of $\underline{\tau}$, is equal to $z_{j} / z_{j}^{1}$, $j=1,2, \ldots, k$ and thus $\underline{\tau} \in(0,1]^{k}$. By (A1), there exists some strictly increasing transformations $F^{*}$ such that

$$
P\left(X^{1} ; \underline{e}_{k}\right)=F^{*}\left[P\left(X^{1} ; \underline{\tau}\right), \underline{e}_{k}, \underline{\tau}\right]:=F\left[P\left(X^{1} ; \underline{\tau}\right), \underline{\tau}\right], \quad \underline{\tau} \in(0,1]^{k}
$$

where $F[., \underline{\tau}]:=F^{*}\left[., \underline{e}_{k}, \underline{\tau}\right]$. Since $P\left(X^{0} ; \underline{e}_{k}\right)=P\left(X^{1} ; \underline{\tau}\right)$ by RS, where the $i j^{\text {th }}$ element of $X^{0}$ is $x_{i j} / z_{j}$, (A2) may also be expressed as:

$$
P\left[X^{1} ; \underline{e}_{k}\right]=F\left[P\left(X^{0} ; \underline{e}_{k}\right) ; \underline{\tau}\right] .
$$

Let $\theta_{i j}:=x_{i j} / z_{j}, \quad \theta_{i j}^{1}:=x_{i j} / z_{j}^{1}, \quad j=1,2, \ldots, k, \quad \underline{\theta}_{i}:=\left(\theta_{i 1}, \ldots, \theta_{i k}\right), \quad \underline{\theta}_{i}^{1}:=$ $\left(\theta_{i 1}^{1}, \ldots, \theta_{i k}^{1}\right)$. Using Eq. (1) in the text and (A3):

$$
G\left[\frac{1}{n} \sum_{i=1}^{n} \phi\left(\underline{\theta}_{i}^{1}, \underline{e}_{k}\right) ; \underline{e}_{k}\right]=F\left\{G\left[\frac{1}{n} \sum_{i=1}^{n} \phi\left(\underline{\theta}_{i}, \underline{e}_{k}\right) ; \underline{e}_{k}\right] ; \underline{\tau}\right\},
$$

for all $\theta_{i} \in(0,1]^{k}$ such that $\underline{\theta}_{i}^{1}=\underline{\theta}_{i} \Gamma, \Gamma:=\operatorname{diag}(\underline{\tau}), \underline{\tau} \in(0,1]^{k}$. The above equation may be rewritten as:

$$
G^{*}\left[\frac{1}{n} \sum_{i=1}^{n} \phi^{*}\left(\underline{\theta}_{i}^{1}\right)\right]=F\left[G^{*}\left(\frac{1}{n} \sum_{i=1}^{n} \phi^{*}\left(\underline{\theta}_{i}\right)\right), \underline{\tau}\right], \quad \underline{\theta}_{i}, \underline{\theta}_{i}^{1}, \underline{\tau} \in(0,1]^{k}
$$

where $G^{*}[u]=G\left[u, \underline{e}_{k}\right], \phi^{*}(u)=\phi\left(u, \underline{e}_{k}\right)$, Let $\underline{\theta}_{i}=\underline{\theta}$ and so $\underline{\theta}_{i}^{1}=\underline{\theta} \Gamma=\underline{\theta}^{1}$ for all $i$. Inverting the term on the left hand side of Eq. (A4) with respect to $G^{*}$ and setting $\underline{\theta}_{i}^{1}=\underline{\theta}^{1}$, Eq. (A4) becomes

$$
\phi^{*}\left(\underline{\theta}^{1}\right)=G^{*-1}\left[F\left(G^{*}\left(\phi^{*}(\underline{\theta})\right), \underline{\tau}\right)\right]:=H\left[\phi^{*}(\underline{\theta}), \underline{\tau}\right] .
$$

Let $n=2 m$ with $m$ persons having $\underline{\theta}_{i}=\underline{\theta}, \underline{\theta}_{i}^{1}=\underline{\theta}^{1}$ and the other $m$ persons with $\underline{\theta}_{i}=\underline{\sigma}, \underline{\theta}_{i}^{1}=\underline{\sigma}^{1}$. By (A4),

$$
G^{*}\left[\frac{\phi^{*}\left(\underline{\theta}^{1}\right)+\phi^{*}\left(\underline{\sigma}^{1}\right)}{2}\right]=F\left[G^{*}\left(\frac{\phi^{*}(\underline{\theta})+\phi^{*}(\underline{\sigma})}{2}\right), \underline{\tau}\right]
$$

Let $\phi^{*}(\underline{\theta}):=u$ and $\phi^{*}(\underline{\sigma}):=v$ and using (A5),

$$
\frac{H(u, \underline{\tau})+H(v, \underline{\tau})}{2}=H\left(\frac{u+v}{2}, \underline{\tau}\right), \quad u, v \in R g \phi^{*} .
$$

where $R g \phi^{*}$ is the range of $\phi^{*}$. The above is a Jensen equation whose solution is (Aczel 1966, p. 44-46):

$$
H[u, \underline{\tau}]=\alpha(\underline{\tau}) u+\beta(\underline{\tau}) .
$$


By (A5) and the definition of $u$,

$$
\phi^{*}\left(\underline{\theta}^{1}\right)=\alpha(\underline{\tau}) \phi^{*}(\underline{\theta})+\beta(\underline{\tau}) .
$$

Since $\theta_{i}^{1}=\underline{\theta}_{i} \Gamma, \Gamma:=\operatorname{diag}(\underline{\tau}), \underline{\tau} \in(0,1]^{k}$. it follows that Eq. (A7) may be written as

$$
\phi^{*}(\underline{\theta} \Gamma)=\alpha(\underline{\tau}) \phi^{*}(\underline{\theta})+\beta(\underline{\tau}), \quad \underline{\theta}, \underline{\tau}, \underline{\theta} \Gamma \in(0,1]^{k} .
$$

Without the domain restrictions on $\underline{\theta}$ and $\underline{\tau}$, the solution of (A8) is well-known (Aczel et al. 1986). It is straightforward to show that the solution for (A8) on the present restricted domain is:

$$
\phi^{*}(\underline{\theta})=\left\{\begin{array}{l}
a \prod_{j=1}^{k} \theta_{j}^{r_{j}}+b \\
\sum_{j=1}^{k} c_{j} \log \theta_{j}+d
\end{array}, \quad \underline{\theta} \in(0,1]^{k}\right.
$$

where $a, b, d, r_{j}$ and $c_{j}$ are parameters. Since $\phi^{*}\left(\underline{e}_{k}\right)=0$ (see Proposition 1), $b=-a$ and $d=0$.

So far, the domain of $\phi^{*}(\underline{\theta})$ is restricted to $\underline{\theta} \in(0,1]^{k}$. For $\underline{\theta} \in[1, \infty)^{k}$, $\phi^{*}(\underline{\theta})=0$. With regard to the the functional forms in those domains when $\theta_{j}>1$, for some $j$, let $\hat{\theta}:=\left\{\hat{\theta}_{1}, \ldots, \hat{\theta}_{k}\right\}$, where $\hat{\theta}_{j}=\min \left\{\theta_{j}, 1\right\}$. Then, $\phi^{*}(\underline{\theta})=\phi^{*}(\underline{\hat{\theta}})$. Thus, the forms of $\phi^{*}(\underline{\theta})$ with respect to the entire domain $\mathbb{R}_{++}^{k}$ is ascertained. Substituting the functional forms of $\phi^{*}$ into $P(X ; \underline{z})$, we arrive at the following expression:

$$
P(X ; \underline{z})=\left\{\begin{array}{l}
G\left[\frac{a}{n} \sum_{i=1}^{n}\left[\prod_{j=1}^{k}\left(\frac{x_{i j} \wedge z_{j}}{z_{j}}\right)^{r_{j}}-1\right] ; \underline{e}_{k}\right] \\
G\left[\frac{1}{n} \sum_{i=1}^{n} \sum_{j=1}^{k} c_{j} \log \left(\frac{x_{i j} \wedge z_{j}}{z_{j}}\right) ; \underline{e}_{k}\right]
\end{array}\right.
$$

Since $\phi^{*}(\underline{\theta})=\phi^{*}(\underline{\hat{\theta}})$, this explains why the term $x_{i j} \wedge z_{j}$ appears in the functional forms above. Since $a r_{i} \leq 0$ for all $i$ by $\mathrm{MN}$ and $a r_{i} r_{j} \geq 0, i \neq j$, for $\phi^{*}$ to be L-superadditive on $\mathfrak{P}$, it immediately follows that $r_{j} \leq 0$, for all $j$, and $a>0$. Furthermore, there is at least one $r_{j}$ not equal to zero because the index is nontrivial. Furthermore, the paramters are to assume values such that $\phi^{*}(\underline{\theta})$ should be convex.

Let $\alpha_{j}=-r_{j}, \delta_{j}=-c_{j} / a$ and the increasing transformation of $P$ be $G^{-1}\left[., \underline{e}_{k}\right] / a$, then we arrive at Eqs. (3a) and (3b) together with the restrictions on the parameters.

The proof of the converse is straightforward.

Q.E.D.

Proof of Proposition 6. Let us first restrict the domain of $P$ to $\mathfrak{M}(z) \times Z$ where $\mathfrak{M}(\underline{z}):=\left\{X \in \mathfrak{M}: x_{i j} \leq z_{j}\right.$ for all $i$ and $\left.j\right\}$. Define a signs sequence $\sigma:=$ $\left\{\sigma_{1}, \ldots, \sigma_{k}\right\}$ as follows: 


$$
\sigma_{j}=\left\{\begin{array}{ll}
+1, & x_{i j} \in \mathbb{R}++ \\
-1, & x_{i j} \in \mathbb{R}-
\end{array} .\right.
$$

Each $\sigma_{j}$ indicates whether the value of the $j$ th attribute is restricted to $\mathbb{R}_{++}$or $\mathbb{R}_{-}{ }^{23}$ For any given $k$, there are altogether $2^{k}$ signs sequences. For any given signs sequence, let $\mathbb{R}_{\sigma}^{k}$ be an orthant with the domain restrictions on the $k$ attributes as indicated by $\sigma$. Then $\mathscr{E}:=\left\{\mathbb{R}_{\sigma}^{k}\right.$ : all possible signs sequences $\left.\sigma\right\}$ is a partition of $\mathbb{R}^{k} ;{ }^{24} \mathfrak{M}_{\sigma}$ is a subset of $\mathfrak{M}$ with the domain restrictions on the $k$ attributes as specified by $\sigma$. Correspondingly, $\mathscr{F}:=\left\{\mathfrak{M}_{\sigma}\right.$ : all possible signs sequence $\sigma\}$ is a partition of $\mathfrak{M}$.

The poverty measure continues to have the functional form as depicted by Eq. (1). Furthermore, the proof of Proposition 2 up to Eq. (A7) may be redeployed here to show that $\phi^{*}(\underline{\theta} \Gamma)=\alpha(\underline{\tau}) \phi^{*}(\underline{\theta})+\beta(\underline{\tau})$ though the domain restrictions are now different, i.e., $\theta_{j} \in(-\infty, 0]$ or $\theta_{j} \in(0,1]$. The change in the domain turns out to have a dramatic impact on the functional form of $\phi^{*}($.$) . For some arbitrary \mathfrak{M}_{\sigma} \cap \mathfrak{M}(\underline{z})$, let $\underline{\delta}:=\left(\delta_{1}, \ldots, \delta_{k}\right)$ such that

$$
\delta_{j}=\left\{\begin{array}{ll}
-\theta_{j}, & \theta_{j} \leq 0 \\
\theta_{j}, & \theta_{j}>0
\end{array} \quad j=1,2, \ldots, k .\right.
$$

Since $\theta_{j}$ is either in $(-\infty, 0]$ or in $(0,1]$, the corresponding domain of $\delta_{j}$ is either $[0, \infty)$ or $(0,1]$. Restricted to $\mathbb{R}_{\sigma}^{k}, \phi^{*}(\underline{\theta})=\phi^{*}\left(\sigma_{1} \delta_{1}, \ldots, \sigma_{k} \delta_{k}\right)$; we define $\phi_{\sigma}^{*}(\underline{\delta}):=\phi^{*}\left(\sigma_{1} \delta_{1}, \ldots, \sigma_{k} \delta_{k}\right)$. Then:

$$
\phi_{\sigma}^{*}(\underline{\delta} \Gamma)=\alpha(\underline{\tau}) \phi_{\sigma}^{*}(\underline{\delta})+\beta(\underline{\tau}),
$$

where $\underline{\tau} \in(0,1]^{k}, \Gamma:=\operatorname{diag}(\underline{\tau}), \delta_{j} \in(0,1]$ when $\sigma_{j}=+1$ or $\delta_{j} \in[0, \infty)$, when $\sigma_{j}=-1, j=1,2, \ldots, k$. While the solution of the above functional equation is well-known when the domain is $[0, \infty)^{k}$, it can be shown that, for some given $\sigma$, the solution with the above domain restrictions is,

$$
\phi_{\sigma}^{*}(\underline{\delta})=\left\{\begin{array}{l}
a_{\sigma} \prod_{j \in \Omega_{\sigma}} \delta_{j}^{r_{\sigma j}}+b_{\sigma} \\
\sum_{j \in \Lambda_{\sigma}}^{k} c_{\sigma j} \ell n \delta_{j}+d_{\sigma}
\end{array}\right.
$$

where $\Omega_{\sigma}:=\left\{j \in\{1,2 \ldots, k\}: \delta_{j} \in[0, \infty)\right.$ and $\left.\alpha\left(, 1,1, \ldots, \tau_{j}, \ldots, 1\right) \neq 1\right\} \cup$ $\left\{j \in\{1,2, \ldots, k\}: \delta_{j} \in(0,1]\right\}, \Lambda_{\sigma}:=\left\{j: \delta_{j}>0, \underline{\delta} \in \mathbb{R}_{\sigma}^{k}\right\}$ and

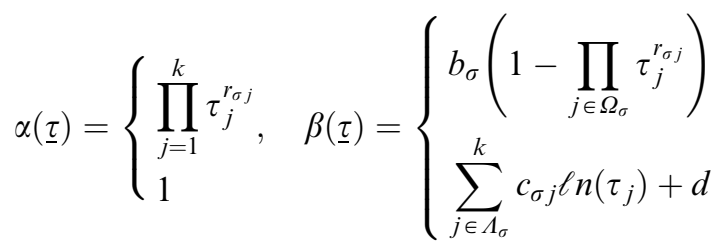

${ }^{23}$ For example, when $k=3$, an example of a signs sequence is $\{+1,-1,+1\}$.

${ }^{24}$ When $k=2, \mathscr{E}=\{\{+1,+1\},\{+1,-1\},\{-1,+1\},\{+1,+1\}\}$. 
It is to be noted that $\delta_{j}=\left|\theta_{j}\right|$. The above solution is a straightforward but tedious extension of the standard result without the normal domain restrictions. Details of the proof are avaiblable on request.

Since $\alpha($.$) and \beta($.$) are independent of \sigma, b_{\sigma}=b$ and $r_{\sigma j}=r_{j}, c_{\sigma j}=c_{j}$, $d_{\sigma}=d$, for all $\sigma$. Fitting $\left\{\phi_{\sigma}^{*}\right\}$ together, we can derive $\phi^{*}$ on $\mathbb{R}^{k}=\bigcup_{\sigma}\left\{\mathbb{R}_{\sigma}^{k}\right\}$. For $\phi^{*}$ to be continuous on $\bigcup_{\sigma}\left\{\mathbb{R}_{\sigma}^{k}\right\}$, the log-linear form is eliminated and we are left with

$$
\phi^{*}(\underline{\theta})=a_{\sigma} \prod_{j \in \Omega_{\sigma}}\left|\theta_{j}\right|^{r_{j}}+b
$$

where $\underline{\theta} \in \mathbb{R}_{\sigma}^{k}$. The above proof is available on request. So far the proof is restricted to $\mathfrak{M}(\underline{z})$. By Proposition $1, \phi^{*}(\underline{\theta})=\phi^{*}(\underline{\hat{\theta}})$ where $\underline{\hat{\theta}}=\left\{\underline{\theta}_{1}, \ldots, \underline{\hat{\theta}}_{k}\right\}$, $\hat{\theta}_{j}=\min \left\{\theta_{j}, 1\right\}$ for any $\underline{\theta}$. Thus, we have identified the function form of $\phi^{*}$ on $\mathfrak{M}$.

It can also be show that $r_{j} \geq 0$ for all $j$, with $r_{j}>0$ for at most one $j$. Without loss in generality, let $\sigma$ be a signs sequence such that $\sigma_{j}=+1$, for all $j$; then, $a_{\sigma}=-b \leq 0$ because $b=\phi_{\sigma}^{*}(\underline{0}) \geq \phi_{\sigma}^{*}\left(\underline{e}_{k}\right)=0$ by $\mathrm{MN}$; hence $r_{j} \geq 0$, for all $j$ by MN. Let $\pi$ be another signs sequence with $\pi_{\ell}=-1$ and $\pi_{j}=\sigma_{j}$ for $j \neq \ell$. Then, for any $\underline{\theta} \in \mathbb{R}_{\pi}^{k}, \theta_{\ell}<0$ and $\theta_{j} \geq 0$ for $j \neq \ell$. Furthermore, $r_{\ell} \geq 0$. By $\mathrm{MN}, a_{\pi} \geq 0$ because $\theta_{\ell}<0$. At the same time, with $r_{j} \geq 0$ and $\theta_{j} \geq 0, j \neq \ell, a_{\pi} \leq 0$. As a corollary, either $a_{\pi}=0$ or there can at most be one $r_{j}$ positive, say, $r_{m}$, such that

$$
\phi^{*}(\underline{\theta})=\left\{\begin{array}{l}
b\left(1-\theta_{m}^{r_{m}}\right), \quad 0<\theta_{m} \leq 1, \\
b\left(1+\left(a_{\sigma} / b\right)\left|\theta_{m}\right|^{r_{m}}\right), \quad \theta_{m} \leq 0,
\end{array}\right.
$$

where $b>0, r_{m}>0$ and $a_{\sigma} \geq 0$. Furthermore, by Propositions 2 the parameters in (A13) have to be restricted in such a way that $\phi^{*}$ is convex, implying that $r_{m}$ must be equal to one and $a_{\sigma} / b \geq 1$. By Proposition $3, \phi^{*}$ has to be L-superadditive and this is trivially satisfied by (A13). Tranforming $P$ by $G^{-1}\left[. ; \underline{e}_{k}\right] / b$ and letting $a$ be $-a_{\sigma} / b$, we arrive at Eq. (4) in the text.

The converse of the proof is straightforward.

Q.E.D.

Proof of Lemma 1. Foster and Shorrocks (1991) provide the proof for the case with one attribute. The proofs of the multidimensional cases are similar and are therefore not presented here. The proofs, however, may be provided on request.

Q.E.D.

Proof of Proposition 7. With regard to case (a), let us as before restrict the domain of $P$ to $\mathfrak{M}^{++}(\underline{z}) \times \mathbb{R}_{++}^{k}$. Invoking Lemma 1 and PCI, there exists $\hat{F}$ such that

$$
\hat{G}_{A}\left[\frac{1}{n} \sum_{i=1}^{n} \psi\left(\underline{w}_{i}^{1}\right) ; \underline{h}\right]=\hat{F}\left\{\hat{G}_{A}\left[\frac{1}{n} \sum_{i=1}^{n} \psi\left(\underline{w}_{i}\right) ; \underline{0}_{k}\right] ; \underline{h}\right\}
$$

where $\hat{G}_{A}[., \underline{h}]:=G_{A}[., \underline{z}+\underline{h}], \hat{F}[., \underline{h}]:=F[., \underline{z}, \underline{z}+\underline{h}], \underline{w}_{i}=\underline{z}-x_{i}, \underline{w}_{i}^{1}=\underline{w}_{i}+$ $\underline{h} \in \prod_{j}\left[0, z_{j}\right), \underline{h} \in \mathbb{R}_{+}^{k}$. Let $\underline{w}_{i}=\underline{u}$ for all $i$ and $\underline{u}^{1}:=\underline{u}+\underline{h}$. Then, 


$$
\psi\left(\underline{u}_{1}\right)=\hat{G}_{A}^{-1}\left\{\hat{F}\left\{\hat{G}_{A}\left[\psi(\underline{u}) ; \underline{0}_{k}\right] ; \underline{h}\right\} ; \underline{h}\right\}:=\hat{H}[\psi(\underline{u}) ; \underline{h}]
$$

Next, let $n=2 m$ such that $\underline{w}_{i}=\underline{u}, \quad i=1,2, \ldots, m$ and $\underline{w}_{i}=\underline{v}$, $i=m+1, \ldots, n$,

$$
\hat{G}_{A}\left[\frac{1}{2} \psi\left(\underline{u}^{1}\right)+\frac{1}{2} \psi\left(\underline{v}^{1}\right) ; \underline{h}\right]=\hat{F}\left\{\hat{G}_{A}\left[\frac{1}{2} \psi(\underline{u})+\frac{1}{2} \psi(\underline{v}) ; \underline{0}_{k}\right] ; \underline{h}\right\}
$$

where $\underline{u}^{1}:=\underline{u}+\underline{h}$ and $\underline{v}^{1}:=\underline{v}+\underline{h}$. Using (A15) and (A16):

$$
\frac{\hat{H}(s, \underline{h})+\hat{H}(t, \underline{h})}{2}=\hat{H}\left[\frac{s+t}{2} ; \underline{h}\right], \quad s, t \in R g \psi
$$

where $\psi(\underline{u}):=s$ and $\psi(\underline{v}):=t$ and $R g \psi$ is the range of $\psi$. The above is a Jensen equation with the following solution:

$$
\hat{H}(s, \underline{h})=\alpha(\underline{h}) s+\beta(\underline{h}), \quad \underline{h} \in \mathbb{R}_{+}^{k}, s \in R g \psi .
$$

By (A15) and $\underline{w}^{1}=\underline{w}+\underline{h}$, the above may be rewritten as:

$$
\psi(\underline{w}+\underline{h})=\alpha(\underline{h}) \psi(\underline{w})+\beta(\underline{h}), \quad \underline{w}, \underline{w}+\underline{h} \in \prod_{j}\left[0, z_{j}\right), \underline{h} \in \mathbb{R}_{+}^{k} .
$$

Then the solution of the above functional equation is:

$$
\psi(\underline{w})=\left\{\begin{array}{l}
\alpha \exp \left\{\sum_{j=1}^{k} r_{j} w_{j}\right\}+\beta \\
\sum_{j=1}^{k} \gamma_{j} w_{j}+\delta
\end{array}, \quad \underline{w} \in \prod_{j=1}^{k}\left[0, z_{j}\right) .\right.
$$

Since $\psi(\underline{0})=0$, it follows that $\beta=-\alpha$ and $\delta=0$. By MN, $\alpha r_{j} \geq 0$ and $\gamma_{j} \geq 0$. Because the index is nontrivial, $\alpha>0$ and $r_{j} \geq 0$ by MN with at least one $r_{j}$ strictly positive. There is at least one $\gamma_{j}$ strictly positive because of nontriviality. Furthermore, PNMT(UM) or PNMT(UPD) restrict $\psi(\underline{z}-\underline{u})$ to be convex with respect to $\underline{u}$. By PNR, $r_{i} r_{j} \geq 0$ and this inequality is obviously compatible with other restrictions on the parameters.

So far, (A18) holds for $x_{i j} \leq \underline{z}_{j}$ for all $j$. By Lemma $1, \psi\left(\underline{z}-\underline{x}_{i}\right)=$ $\psi\left(\underline{z}-\underline{x}_{i} \wedge \underline{z}\right)$; furthermore, $\psi\left(\underline{z}-\underline{x}_{i}\right)=0$ when $x_{i j} \geq z_{j}$ for all $j$. We can thus derive the functional form of $\psi$ on the entire domain. Let $c_{j}:=\gamma_{j} / \alpha$, and tranforming $P$ by $\alpha^{-1} G_{A}^{-1}(. ; z)$, we arrive at Eqs. (6a) and (6b) in the text.

The proof above can also be applied to case (b). Equation (A14) is still valid and the domain restrictions become $\underline{w}, \underline{w}+\underline{h} \in \mathbb{R}_{+}^{k}, \underline{h} \in \mathbb{R}_{+}^{k}$. It can be shown (despite the change in domain restrictions) that the solution is still (A18). The proof, which is similar to the well known case with normal restrictions, is available on demand. For case (c), we may transform $X$ and $\underline{z}$ such that $u_{i j}:=\exp \left(x_{i j}\right)$ and $v_{j}:=\exp \left(z_{j}\right)$. Then the poverty measure becomes ratio-scale invariant with respect to the transformed variables. Using Proposition 5, we can show that the class of poverty measures must be (6a) and (6b). Details are available on request.

Q.E.D. 
Proof of Proposition 8. For $X \in \mathfrak{M}^{++}$and $\underline{z} \in \mathbb{R}_{++}^{k}$, the proof is a straightforward adaptation of the one for the unidimensional case in Foster and Shorrocks (1991). By SCM,

$$
P^{\prime}(X ; \underline{z})=\bar{\Gamma}[P(X ; \underline{z}) ; \underline{z}], \quad X, Y \in \mathfrak{M}^{++}, \underline{z} \in \mathbb{R}_{++}^{k} .
$$

For $n=2$, using (1) and (5) in the text,

$$
\frac{1}{2}\left\{\psi\left(\underline{z}-\underline{x}_{1}\right)+\psi\left(\underline{z}-\underline{x}_{2}\right)\right\}=\Gamma\left[\frac{1}{2}\left\{\phi^{*}\left(\underline{x}_{1} \Sigma\right)+\phi^{*}\left(\underline{x}_{2} \Sigma\right)\right\} ; \underline{z}\right]
$$

where $\Gamma=G_{A}^{-1} \circ \bar{\Gamma} \circ G, \Sigma:=\operatorname{diag}\left(z_{1}^{-1}, \ldots, z_{k}^{-1}\right)$ and $\phi^{*}(\underline{\theta}):=\phi\left(\underline{\theta} ; \underline{e}_{k}\right)$; furthermore, if $\underline{x}_{i}=\underline{v}$, then

$$
\psi(\underline{z}-\underline{v})=\Gamma\left[\phi^{*}(\underline{v} \Sigma) ; \underline{z}\right] .
$$

Substituting (A20) into (A19):

$$
\frac{1}{2}\left\{\Gamma\left[\phi^{*}\left(\underline{x}_{1} \Sigma\right) ; \underline{z}\right]+\Gamma\left[\phi^{*}\left(\underline{x}_{2} \Sigma\right) ; \underline{z}\right]\right\}=\Gamma\left[\frac{\phi^{*}\left(\underline{x}_{1} \Sigma\right)+\phi^{*}\left(\underline{x}_{2} \Sigma\right)}{2} ; \underline{z}\right]
$$

The above is a Jensen equation whose solution is $\Gamma(u ; \underline{z})=c(\underline{z}) u$ as $\Gamma(0 ; \underline{z})$ $=0$ with $c(\underline{z})>0$, or

$$
\psi(\underline{z}-\underline{v})=c(\underline{z}) \phi^{*}(\underline{v} \Sigma), \quad \underline{v}, \underline{z} \in \mathbb{R}_{++}^{k} ;
$$

Let $\underline{\theta}:=\underline{e}_{k}-\underline{v} \Sigma$, and $\Theta:=\operatorname{diag}(\underline{\theta})$, then

$$
\psi(\underline{z} \Theta)=c(\underline{z}) \phi^{*}\left(\underline{e}_{k}-\underline{\theta}\right), \quad \underline{z} \in \mathbb{R}_{++}^{k}, \underline{\theta} \in[0,1)^{k} .
$$

The solution of the above domain-restricted functional equation is that ${ }^{25}$

$$
\psi(\underline{u})=\eta_{1} \prod_{j=1}^{k} u_{j}^{\alpha_{j}}, \quad c(\underline{z})=\eta_{2} \prod_{j=1}^{k} z_{j}^{\alpha_{j}}, \quad \phi^{*}(\underline{v})=\eta_{3} \prod_{j=1}^{k}\left(1-v_{j}\right)^{\alpha_{j}}
$$

for $\underline{u} \in \mathbb{R}_{++}^{k}, \underline{z} \in \mathbb{R}_{++}^{k}$ and $\underline{v} \in(0,1]^{k}$. The corresponding class of relative measures is:

$$
P(X ; \underline{z})=G\left[\frac{\eta_{3}}{n} \sum_{i=1}^{n} \prod_{j=1}^{k}\left(1-\frac{x_{i j} \wedge z_{j}}{z_{j}}\right)^{\alpha_{j}} ; \underline{z}\right] .
$$

Furthermore, $\eta_{1}, \eta_{2}$ and $\alpha_{j}$ are all nonnegative because of MN. By nontriviality and $\mathrm{CN}$, these parameters are positive. By $\mathrm{PNMT}(\mathrm{UM})$ (or PNMT(UPD)) and PNR, $\phi^{*}$ have to be convex and L-superadditive. Of all the restrictions on $\phi^{*}$ to ensure convexity, two of them are

$$
\phi_{11}^{*} \geq 0, \quad \phi_{11}^{*} \phi_{22}^{*}-\phi_{12}^{* 2} \geq 0 .
$$

25 This is a Pexider equation with several variables. The solution when the variables are positive is well-known. However, in our case, $\underline{\alpha} \in[0,1)^{k}$. Yet, it is not difficult to shown that the solution is the same. 
They imply that $\eta_{3} \alpha_{1}\left(\alpha_{1}-1\right) \geq 0$ and $1-\alpha_{1}-\alpha_{2} \geq 0$. By MN, $\eta_{3} \alpha_{i}$ is positive for all $i$, thus $\alpha_{i}-1 \geq 0$, implying that the inequality $1-\alpha_{1}-\alpha_{2} \geq 0$ can never be satisfied.

With regard to the translation-scale invariant index:

$$
P(X ; \underline{z})=G\left[\frac{\eta_{1}}{n} \sum_{i=1}^{n} \prod_{j=1}^{k}\left(z_{j}-x_{j}\right)^{\alpha_{j}} ; \underline{z}\right] \text {. }
$$

By a similar argument, it can be shown that the absolute index cannot satisfy all the axioms.

Q.E.D.

\section{References}

Aczel J (1966) Lectures on functional equations and their applications. Academic Press, New York, London

Aczel J (1987) A short sourse on functional equations. D. Reidel Publishing Company, Dordrecht, Holland

Aczel J, Roberts F, Rosenbaum Z (1986) On scientific laws without dimensional constants. J Math Analy Appl 119: 389-416

Atkinson AB (1987) On the measurement of poverty. Econometrica 55: 749-764

Atkinson AB (1989) Poverty and social security. Harvester Wheatsheaf, New York

Atkinson AB (1992) Measuring poverty and differences in family composition. Economica 59: $1-16$

Atkinson AB, Bourguignon F (1982) The comparison of multi-dimensioned distributions of economic status. Rev Econ Stud 49: 183-201

Berhman J, Deolalikar AB (1988) Health and nutrition. In: Chenery H, Srinivasan TN (eds.), Handbook of development economics. Elsevier Science Publishers, Amsterdam

Blackorby C, Russell RR (1980) Ethical indices for the measurement of poverty. Econometrica 48: 1053-1060

Borland PJ, Proschan F (1988) Multivariate arrangement increasing functions with applications in probability and statistics. J Multivariate Anal 25: 286-298

Bossert W, Pfingsten A (1990) Intermediate inequality: concepts, indices, and welfare implications. Math Soc Sci 19: 117-134

Bourguignon F, Chakravarty SR (1997a) A family of multidimensional poverty measures. mimeo

Bourguignon F, Chakravarty SR (1997b) The measurement of multidimensional poverty. mimeo

Callan T, Nolan B (1991) Concepts of poverty and the poverty line. J Econ Surveys 5: 243-261

Chakravarty SR (1983) A new index of poverty. Math Soc Sci 6: 307-313

Chakravarty SR (1990) Ethical social index numbers. Springer, Berlin Heidelberg New York

Chakravarty SR, Mukherjee D, Ranade RR (1998) On the family of subgroup and factor decomposable measures of multidimensional poverty. Res Econ Inequality 8: $175-194$

Dardanoni V (1995) On multidimensional inequality measurement. Res Econ Inequality 6: 201-207

Dasgupta P (1993) An inquiry into well-being and destitution. Clarendon Press, Oxford

Dasgupta P, Sen AK, Starrett D (1973) Notes on the measurement of inequality. J Econ Theory 6: 180-187 
Datt G, Ravallion M (1992) Growth and redistribution components of changes in poverty measures: a decomposition with applications to Brazil and India in the 1980s. J Develop Econ 38: 275-295

Donaldson D, Weymark JA (1986) Properties of fixed-population poverty indices. Inte Econ Rev 27: 667-688

Dreze J, Sen A (1989) Hunger and public action. Clarendon Press, Oxford

Foster J (1984) On economic poverty: a survey of aggregative measures. Adv Econ 3: $215-251$

Foster J, Greer J, Thorbecke E (1984) A class of decomposable poverty measures. Econometrica 52: 761-766

Foster J, Shorrocks A (1988a) Poverty orderings. Econometrica 56: 172-177

Foster J, Shorrocks A (1988b) Poverty orderings and welfare dominance. Soc Choice Welfare 5: 179-198

Foster J, Shorrocks A (1991) Subgroup consistent poverty indices. Econometrica 59: $687-709$

Hirschberg J, Maasoumi E, Slottje D (1991) Cluster analysis for measuring welfare and quality of life across countries. J Econ 50: 131-150

Joe H, Verducci J (1993) Multivariate majorization by positive combinations. In: Stochastic inequalities (IMS Lecture Notes - Monograph Series, 22) pp. 159-181

Kakwani N (1984) Issues in measuring poverty. Adv Econ 3: 253-282

Kanbur SMR (1987) Measurement and alleviation of poverty. IMF Staff Papers, pp. $60-85$

Kolm S-Ch (1976a) Unequal inequalities I. J Econ Theory 12: 416-42

Kolm S-Ch (1976b) Unequal inequalities II. J Econ Theory 13: 82-111

Kolm S-Ch (1977) Multidimensional egalitarianisms. Q J Economics 91: 1-13

Koshevoy G (1995) Multivariate Lorenz Majorization. Soc Choice Welfare 12: 93102

Koshevoy G, Mosler K (1996) The Lorenz surface of a multivariate distribution. J Am Stat Assoc 91: 873-882

Maasoumi E (1989) Composite indices of income and other developmental indicators: a general approach. Res Econ Inequality 1: 269-286

Maasoumi E, Nickelsburg G (1988) Multivariate measures of well-being and an analysis of inequality in the Michigan data. J Bus Econ Stat 6: 327-334

Marshall AW, Olkin I (1979) Inequalities: theory of majorization and its application. Academic Press, New York

Mosler K (1993) Multidimensional welfarisms. In: Eichhorn W (ed.), Models and measurement of inequality and welfare. Springer, Berlin Heidelberg New York

Mosler K (1994) Majorization in economic disparity measures. Lin Algebra Its Appl 199: $91-114$

Ram R (1982) Composite indices of physical quality of life, basic needs fulfilment, and income: a 'principle component' representation. J Develop Econ 11: 227-247

Ravallion M (1990) Income effects on nutrition. Econ Develop Cultural Change 38: 489-516

Ravallion M (1996) Issues in measuring and modelling poverty. Econ J 106: 1328 1343

Ravallion M (1992) On 'Hunger and Public Action': a review article on the book by Jean Dreze and Amartya Sen. The World Bank Research Observer 7: 1-16

Seidl C (1988) Poverty measurement: a survey. In: Bos D, Rose M, Seidl C (eds.), Welfare and efficiency in public economics. Springer, Berlin Heidelberg New York

Sen AK (1976) Poverty: an ordinal approach to measurement. Econometrica 44: 219231

Sen AK (1981) Poverty and famine: an essay on entitlement and deprivation. Clarendon Press, Oxford

Sen AK (1985) Commodities and capabilities. North-Holland, Amsterdam

Sen AK (1987) The standard of living. Cambridge University Press, Cambridge 
Sen AK (1988) The concept of development. In: Chenery H, Srinivasan TN (eds.). Handbook of development economics, vol. I Elsevier Science Publishers B.V., Amsterdam, pp. 9-26

Sen AK (1992) Inequality reexamined. Clarendon Press, Oxford

Streeten P (1981) First things first: meeting basic human needs in developing countries. Oxford University Press, New York

Streeten P (1995) Thinking about development. Cambridge University Press, New York

Slottje DJ (1991) Measuring the Quality of Life Across Countries. Rev Econ Stat 73: $684-693$

Tsui K-y (1999) Multidimensional inequality and multidimensional generalized entropy measures: an axiomatic approach. Soc Choice Welfare 16: 145-158

United Nations Development Programme (various issues), Human development report. Oxford University Press, New York

Watts HW (1968) An economic definition of poverty. In: Moynihan DP (ed.), On understanding poverty. Basic Books, New York

Zheng B (1993) An axiomatic characterization of the Watts poverty measures. Econ Lett 42: 81-86

Zheng B (1994) Can a poverty index be both relative and absolute. Econometrica 61: $1453-1458$

Zheng B (1997) Aggregate poverty measures. J Econ Surveys 11: 123-162 